\title{
Aromatase and steroid sulfatase mRNA expression in fine needle aspirates from benign and malignant breast disorders
}

\author{
GAO Lei ${ }^{1}$, QI Ying ${ }^{4}$, LIU JingLi ${ }^{2}$, YU XiaoMeng ${ }^{3}$, XIA Wei $^{4} \&$ LIU GuiJian ${ }^{1 *}$ \\ ${ }^{1}$ Laboratory Department, Guang An'men Hospital, China Academy of Chinese Medical Sciences, Beijing 100053, China; \\ ${ }^{2}$ Clinical Research Institute, Beijing Friendship Hospital, Capital Medical University, Beijing 100050, China; \\ ${ }^{3}$ Department of Pathology, Beijing Friendship Hospital, Capital Medical University, Beijing 100050, China; \\ ${ }^{4}$ Medical Laboratory School, Beihua University, Jilin 132013, China
}

Received November 2, 2011; accepted March 27, 2012

\begin{abstract}
The fine needle aspiration (FNA) test is a convenient and tolerable technique with minimal invasion that is accepted by most women. Local estrogen synthesis depends mainly on the aromatase and steroid sulfatase pathways that are believed to play important roles in breast carcinogenesis. However, little is known about the level of aromatase and steroid sulfatase mRNA expression in FNA samples which contain only small amounts of tissue. The nested Q-PCR assay has been proven to be a highly sensitive and specific method to assess the aromatase expression of breast tissue. In this study, aromatase and steroid sulfatase mRNA expression in 74 patients with benign or malignant disorders was evaluated and compared using nested Q-PCR and non-nested Q-PCR assays. The expression levels were analyzed and correlated with clinical parameters. No difference in the aromatase expression levels between nested and non-nested Q-PCR was noticed. Age and aromatase mRNA expression level were two independent risk factors for breast cancer $(P=0.04$ and $P=0.00$, respectively), while menopausal status and steroid sulfatase mRNA expression levels were not associated with breast cancer. This study showed that both nested and non-nested Q-PCR assays were effective methods for research using FNA breast samples.
\end{abstract}

aromatase, steroid sulfatase, breast disorders, fine needle aspirate, nested Q-PCR

Citation: Gao L, Qi Y, Liu J L, et al. Aromatase and steroid sulfatase mRNA expression in fine needle aspirates from benign and malignant breast disorders. Chin Sci Bull, 2012, 57: 2574-2579, doi: 10.1007/s11434-012-5265-1

A variety of evidence suggests that estrogen is critical in breast carcinogenesis [1-3]. Intratumoral estrogen concentrations in breast cancer are much higher than in plasma, especially in postmenopausal women, indicating that local estrogen production might be a major contributor [4]. The difference between plasma and tissue estrogen levels may be caused by the aromatase and sulfatase enzymes that are active in two local estrogen biosynthesis pathways in breast tissue [5]. Aromatase and steroid sulfatase expressions were considered as markers of endocrine responsiveness which may have prognostic implications for breast cancer progression [6].

Recently, fine needle aspiration (FNA) has been used for

*Corresponding author (email: liuguijian@ hotmail.com) cytological assessment and identification of surrogate molecular markers. This method might also aid in predicting breast cancer risk and treatment response [7]. A screening method using this technique would make it possible to investigate normal or pre-malignant breast tissue. Because of a very small amount of breast tissue obtained by FNA, the challenge is to develop a method that is sensitive and specific enough to detect the mRNA expression levels of aromatase and steroid sulfatase. Previously, we established a nested Q-PCR assay for human aromatase and successfully analyzed several ductal lavage fluid samples [8].

In the present study, both aromatase and steroid sulfatase mRNA levels were measured in breast tissues from patients with benign and malignant breast disorders using a nested Q-PCR assay. The aim was to validate the effectiveness of 
the method on FNA samples and to provide new insights into the roles of the estrogen biosynthesis related enzymes in Chinese women with benign and malignant breast disorders.

\section{Materials and methods}

\subsection{Patients}

FNA biopsy samples were collected from 74 patients with a mean age of 48 years (range 22-74) who presented at the Guang An'men and Beijing Friendship hospitals in 2008. The patients were diagnosed with breast cancer or with benign disorders based on a pathohistological examination independently evaluated by two pathologists. At biopsy, none of the patients received treatment for any breast diseases. Clinical data were obtained at the time of examination.

Research protocols for this study were approved by the Ethics Committee at Guang An'men Hospital, affiliated to the China Academy of Chinese Medical Sciences.

\subsection{Preparation of total RNA and cDNA synthesis}

Samples were immediately placed in Qiagen RNAlater RNA Stabilization Reagent until total RNA extraction was performed. Total RNA was extracted from specimens following Bio-Rad PureZol RNA Isolation Reagent protocol. A MMLV Reverse Transcription kit (Invitrogen Co., Carlsbad, CA, USA) was used to synthesize cDNA. Because of the small amount of tissue that could be obtained and based on our experience, a third of the total RNA was used for reverse transcription and RNA concentration determination was omitted.

1.3 Quantitation of aromatase, steroid sulfatase and estrogen receptor $(\mathrm{ER}) \alpha \mathrm{mRNAs}$

Nested two-step PCR reactions were performed using the Light Cycler 480 System (Roche Diagnostics GmbH, Mannheim, Germany). For the first PCR, a master-mix was prepared on ice with $10 \times \mathrm{PCR}$ buffer, $0.6 \mu \mathrm{L}$ of $10 \mathrm{mmol} / \mathrm{L}$ dNTP mix (10 mmol/L each of dATP, dGTP, dCTP and dTTP), $1.6 \mu \mathrm{L}$ of $10 \mathrm{mmol} / \mathrm{L}$ primer (Table 1 ), 1 unit of Qiagen Hot Star Taq DNA polymerase, and $1.2 \mu \mathrm{L}$ of the cDNA template was added to $40 \mu \mathrm{L}$ of the PCR master-mix. The thermal cycling conditions comprised an initial denaturation step at $95^{\circ} \mathrm{C}$ for $15 \mathrm{~min}$ and 20 cycles at $95^{\circ} \mathrm{C}$ for $1 \mathrm{~min}, 54^{\circ} \mathrm{C}$ for $40 \mathrm{~s}$ and $72^{\circ} \mathrm{C}$ for $30 \mathrm{~s}$.

The second step of the nested Q-PCR and non-nested Q-PCR was carried out with the SYBR Green PCR Master Mix kit (Applied Biosystems, USA) using the reaction conditions according to the manufacturer's instructions. An aliquot of $0.5 \mu \mathrm{L}$ of the first PCR product was used as template. The primers used are listed in Table 1.

Relative mRNA expression levels were determined from the amount of TBP mRNA which served as a normalizing gene. Relative mRNA expression $(N)$ was calculated as $N=2^{-\Delta C t}$, where $\Delta C t=C t_{\text {target }}-C t_{\text {reference }}$ and $C t$ is the threshold value during the amplification process.

\subsection{Statistical analysis}

The quantity of relative mRNA expression of the samples showed non-normal distribution, so rank-sum tests were used to examine the relationship between enzyme mRNA

Table 1 Oligonucleotide primer and sequences used in the PCR

\begin{tabular}{|c|c|c|c|}
\hline PCR step & Genes & Sequences & PCR products (bp) \\
\hline \multirow{6}{*}{ Initial PCR } & \multirow{2}{*}{$T B P$} & 5'-AGAACAACAGCCTGCCACCT-3' & \multirow{2}{*}{164} \\
\hline & & 5'-TGCCTTTGTTGCTCTTCCAA-3' & \\
\hline & & 5'-TGGACAGGTTGGAGGAGGTG-3' & \multirow{2}{*}{167} \\
\hline & Aromatase & 5'-GAGAGCTTGCCATGCATCAA-3' & \\
\hline & \multirow{2}{*}{ Steroid sulfatase } & 5'-CTGCTTCATGATGAGGAACT-3' & \multirow{2}{*}{164} \\
\hline & & 5'-TGGAGAACAGGGCTGTGTGC-3' & \\
\hline \multirow{5}{*}{ Nested PCR } & $T B P$ & 5'-CTGAATAGGCTGTGGGGTCA-3' & 115 \\
\hline & Aromotoco & 5'-TGTGGACGTGTTGACCCTTCT-3' & \multirow{2}{*}{93} \\
\hline & Aromatase & 5'-ACCACGATAGCACTTTCGTCCA-3' & \\
\hline & \multirow{2}{*}{ Steroid sulfatase } & 5'-GATCATTCAGCAGCCCATGT-3' & \multirow{2}{*}{115} \\
\hline & & 5'-GAGGTAGGACAAGACAAGCAGG-3' & \\
\hline \multirow{5}{*}{ Non-nested PCR } & \multirow{2}{*}{$T B P$} & 5'-AACAACAGCCTGCCACCTTA-3' & \multirow{2}{*}{115} \\
\hline & & 5'-CTGAATAGGCTGTGGGGTCA-3' & \\
\hline & Aromatase & 5'-ACCACGATAGCACTTTCGTCCA-3' & 93 \\
\hline & \multirow{2}{*}{$E R \alpha$} & 5'-CCACCAACCAGTGCACCATT-3' & \multirow{2}{*}{108} \\
\hline & & 5'-GGTCTTTTCGTATCCCACCTTTC-3' & \\
\hline
\end{tabular}


levels and the clinical parameters of the patients. The correlation between the enzymes and the $E R$ mRNA expression levels was assessed according to the Spearman rank correlation test. The proportional hazards model was used for the multivariate analysis. Statistical significance was assumed for $P<0.05$.

\section{Results}

\subsection{Characteristics of the patients}

All of the collected samples were divided into two categories: cancer group (36 cases) and non-cancer group (38 cases). Clinical characteristics are summarized in Table 2.

\subsection{Validation of the nested Q-PCR assay for FNA sample}

The mRNA expression of aromatase and steroid sulfatase was detected in all the collected FNA samples (74 cases) using both the nested and non-nest Q-PCR. To determine

Table 2 Characteristics of patients

\begin{tabular}{lcc}
\hline & $\begin{array}{c}\text { Cancer group } \\
(n)\end{array}$ & $\begin{array}{c}\text { Non-cancer group } \\
(n)\end{array}$ \\
\hline Age (years) & 10 & 19 \\
$\leqslant 45$ & 12 & 13 \\
$>45 \& \leqslant 55$ & 14 & 6 \\
$>55$ & 18 & \\
\hline Menopausal & 18 & 31 \\
Pre-menopausal & & 7 \\
Post-menopausal & 32 & - \\
\hline Disease status & 4 & - \\
Ductal carcinoma & - & 24 \\
Adenocarcinoma and others & - & 11 \\
Ductal hyperplasia & - & 3 \\
Adenoma & & \\
Others & &
\end{tabular}

the reproducibility and sensitivity of the methods, the aromatase and the TBP housekeeping genes in each sample were measured 3 times on different occasions with both methods. Based on the $C t$ value for each sample during the amplification process, all the samples were assigned a $C t$ value for each of the method and the coefficient of variation $(C V)$ was calculated for each sample. All 74 samples were successfully amplified with the nested Q-PCR assay, and 71 of the 74 samples were successfully amplified with the non-nested Q-PCR assay. For each method, the mean of all the $C V(\%)$ values was calculated. The mean $C V(\%)$ values for the nested and non-nested Q-PCR assays were 2.03 \pm 0.9 and $2.25 \pm 1.5$ respectively. Although the difference in the $C V s(\%)$ was not significant $(P=0.746)$, for 3 FNA samples with trace cells, the nested Q-PCR showed higher sensitivity compared than the non-nested Q-PCR assay.

\subsection{Comparison of aromatase and steroid sulfatase mRNA expression in cancer and non-cancer patients}

As seen in Table 3, aromatase and steroid sulfatase mRNA expression levels were analyzed and all patients were categorized according to the level of enzyme mRNA expression. The enzyme expression levels were compared with the clinical characteristics of the patients. Since mRNA expression quantity data followed a non-normal distribution, the median, minimum and maximum values in each stratum were calculated. The rank-sum tests showed that there were no significant differences in the correlation of aromatase or sulfatase expression with age $(P=0.856$ and $P=0.569$, respectively). However, although the differences in the aromatase mRNA expression level for different age groups were not statistically significant, a trend which showed a growing proportion of samples in the higher age groups with higher expression level of enzyme mRNA could be seen. The mRNA expression level of aromatase correlated with postmenopausal women $(P=0.048)$, but there was no correlation between steroid sulfatase expression and menopausal status $(P=0.529)$.

The aromatase and steroid sulfatase mRNA expression

Table 3 Patients characteristics and mRNA expression of aromatase and steroid sulfatase in all patients with rank-sum tests

\begin{tabular}{|c|c|c|c|c|}
\hline \multirow{3}{*}{ Characteristics } & \multicolumn{4}{|c|}{ Patients mRNA expression } \\
\hline & \multicolumn{2}{|c|}{ Aromatase $^{\mathrm{a})}$} & \multicolumn{2}{|c|}{ Steroid sulfatase ${ }^{\text {b) }}$} \\
\hline & Median (min-max) & $P$ value $^{\mathrm{c})}$ & Median (min-max) & $P$ value $^{\mathrm{c})}$ \\
\hline$\leqslant 45$ & $2.84(0.00-68.87)$ & & $019(0.00-0.66)$ & \\
\hline$>45 \& \leqslant 55$ & $2.84(0.00-115.02)$ & & $0.13(0.10-2.22)$ & \\
\hline$>55$ & $2.78(0.16-201.66)$ & & $0.19(0.03-0.41)$ & \\
\hline Pre-menopausal & $1.62(0.00-115.02)$ & & $0.14(0.00-0.66)$ & \\
\hline Post-menopausal & $5.64(0.02-201.66)$ & & $0.16(0.03-2.22)$ & \\
\hline
\end{tabular}

a) Aromatase mRNA expression level was calculated by using the equation: $N \times 1000, N=2^{-\Delta C t}$; b) steroid sulfatase mRNA expression level was equal to $N, N=2^{-\Delta C t}$; c) $95 \% \mathrm{CI}$, confidence interval. 
levels for the cancer and non-cancer patients were analyzed (Table 4). For the cancer group, the median of the aromatase mRNA expression level was 7.92, compared with 0.44 in the non-cancer group. Aromatase expression was significantly higher in the cancer group than in the non-cancer group $(P<0.001)$. When stratified on menopausal status, no difference in aromatase expression levels was observed when pre- and post-menopausal patients were compared. There was also no significant difference in steroid sulfatase expression levels between the pre- and post-menopausal patients. The relative mRNA expression levels of aromatase and steroid sulfatase in the cancer (malignant) and noncancer groups (benign) are shown in Figure 1. The aromatase relative expression levels differed in benign versus malignant samples although an overlap existed between the two groups (Figure 1(a)). No significant difference was seen in the distribution of steroid sulfatase mRNA expression levels between the malignant and benign groups (Figure 1(b)).

Correlation analysis between the ER $\alpha$ and enzymes mRNA expression levels was also conducted. The Spearman rank correlation test found no correlation between ER $\alpha$ and aromatase $\left(r_{s}=-0.04, P=0.77\right)$ and steroid sulfatase $\left(r_{s}\right.$ $=-0.15, P=0.38$ ) expression levels.

Because the mean age of the patients in the cancer group $(52.7 \pm 9.8)$ was higher than in the non-cancer group $(44.3 \pm$ 10.7 ), we used multivariate analysis to analyze age, menopausal status and enzyme mRNA expression levels in the two groups. Multivariate analysis revealed that age and aromatase mRNA expression were significantly related to the presence of breast cancer $(P=0.04$ and $P=0.00$, respectively); menopausal status and steroid sulfatase mRNA expression level were not associated with breast cancer.

\section{Discussion}

FNA is a breast epithelial sampling technology that provides a new opportunity for breast molecular studies in asymptomatic women $[9,10]$. As only a few cells of breast tissue samples can be obtained by FNA [7], a highly sensitive method for detecting mRNA in the breast tissue aspirates is critical. A sensitive nested Q-PCR developed by us [8] and common non-nested Q-PCR were compared using

Table 4 mRNA expression level of aromatase and steroid sulfatase in cancer and non-cancer group with menopausal status

\begin{tabular}{|c|c|c|c|c|c|}
\hline & & $\begin{array}{c}\text { Cancer group }(n) \\
\text { Median (min-max) }\end{array}$ & $P$ value $^{\mathrm{a})}$ & $\begin{array}{c}\text { Non-cancer group }(n) \\
\text { Median (min-max) }\end{array}$ & $P$ value ${ }^{\text {a) }}$ \\
\hline \multirow{3}{*}{ Aromatase $^{\text {b) }}$} & Menopausal & & 0.602 & & 0.596 \\
\hline & Post-menopausal & $8.00(0.58-201.66)$ & & $0.20(0.02-3.09)$ & \\
\hline & Total & $7.92(0.01-201.66)$ & & $0.44(0.00-115.02)$ & 0.000 \\
\hline \multirow{3}{*}{ Steroid sulfatase ${ }^{c)}$} & Pre-menopausal & $0.17(0.02-0.66)$ & & $0.14(0.00-0.54)$ & \\
\hline & Post-menopausal & $0.17(0.03-2.22)$ & & $0.15(0.01-0.65)$ & \\
\hline & Total & $0.17(0.02-2.22)$ & & $0.14(0.00-0.65)$ & 0.274 \\
\hline
\end{tabular}

a) $95 \% \mathrm{CI}$, confidence interval; b) aromatase mRNA expression level was calculated by using the equation: $N \times 1000, N=2^{-\Delta C t}$; c) steroid sulfatase mRNA expression level was equal to $N, N=2^{-\Delta C t}$.
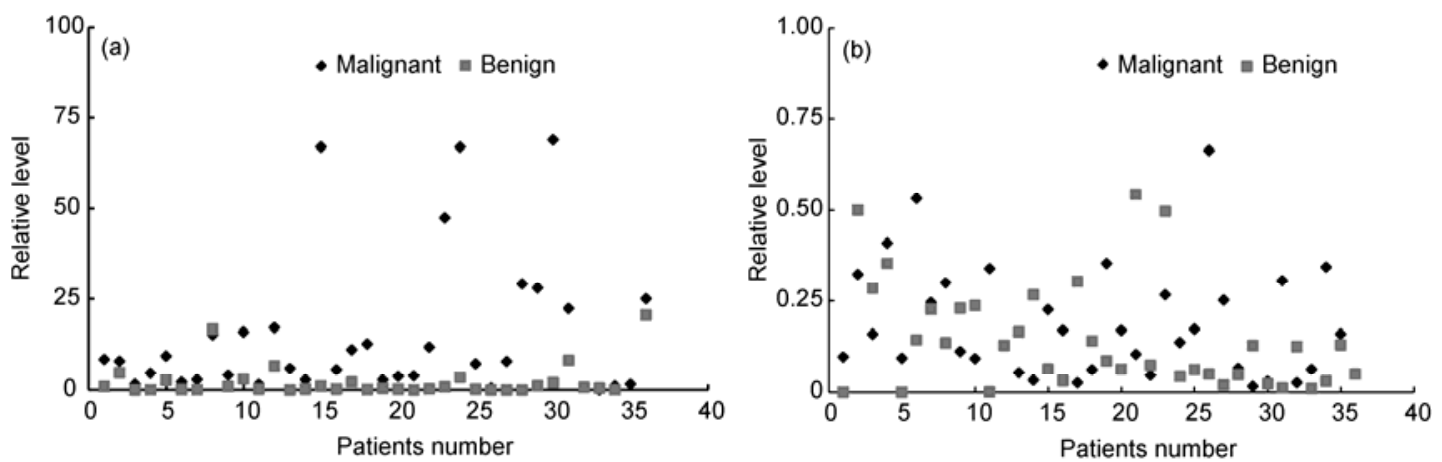

Figure 1 Relative mRNA expression levels of aromatase and steroid sulfatase in cancer (malignant) and non-cancer groups (benign). (a) Aromatase mRNA expression levels in benign and malignant breast cancer. The relative mRNA expression levels were calculated as $N \times 1000$. (b) Steroid sulfatase mRNA expression levels of benign and malignant breast cancer. The relative mRNA expression levels were equal to $N$. $N=2^{-\Delta C t}$, where $\Delta C t=C t_{\text {target }}-C t_{\text {reference }}$ and $C t$ is the threshold value during the amplification process. 
the FNA samples. Our data showed that samples containing trace mRNA were easily detected with nested Q-PCR. Overall there was no obvious difference between the nested and non-nested Q-PCR method; both effectively amplified the aromatase mRNA. This experiment revealed that, for estrogen related enzyme expression studies, the FNA samples were easy to handle. This was in contrast to the results with ductal lavage fluid samples which contain too few cells for valid assay. This study provided additional support for the application of our methodology.

Breast cancer is the major cause of cancer deaths for women in the world [11]. The incidence-age curves of breast cancer around the world are significantly different. In China and Japan, the peak age of breast cancer incidence is about 40-50 years and cancer incidence is associated with increased mortality, while in the United States and other western countries, the incidence continuously increases with age and the morality rate is decreasing $[3,12,13]$. The underlying reasons for the different incidence-age curves are not clear. Many epidemiological and experimental studies showed that estrogens were the fuel behind the aetiology of breast cancer and about $60 \%$ of pre-menopausal and $75 \%$ of post-menopausal patients with breast cancer had estrogen dependent carcinomas [14]. Estrogen synthesis, metabolism, and signaling are considered to be the basic carcinogenic mechanism of breast cancer [15]. The results of this study showed that changes in the peak aromatase and steroid sulfatase mRNA levels had no correlation with the incidence-age curve in China. Therefore, the different incidence-age curves reported in East Asia and western countries need to be studied further.

Cancer tissue and adjacent tissue are generally used in tumor related research. Using Q-PCR analysis, it was confirmed that the adipose stromal cells surrounding cancer cells contained higher levels of aromatase mRNA than adipose stromal cells in non-cancerous areas [16,17]. It was demonstrated that the in situ overexpression of aromatase played an important role in breast tumor promotion [18-20]. In the present study, we collected specimens from patients who underwent FNA cytological test. Therefore, the noncancer specimens we obtained were not tissues neighboring cancer cells, but absolutely "normal" tissue. This allowed us to reliably determine the characteristics of pre-malignant tissue. Most non-cancer patients had breast ductal hyperplasia and some of them had pre-cancerous lesions with varying morphology. Our results showed that, in general, the non-cancer samples expressed lower mRNA levels of aromatase than the cancer samples; however, some noncancer specimens did have very high aromatase expression levels (Figure 1). Breast cancer is the result of a continuous series of events. A possible model of its progression shows its evolution from proliferative disease without atypia (PDWA) to atypical ductal hyperplasia (ADH), from $\mathrm{ADH}$ to ductal carcinoma in situ (DCIS), from DCIS to invasive carcinoma, and finally to invasive ductal carcinoma [20]. In this progression estradiol (E2) has been considered as one of the most risk factors [21,22]. Investigators have shown that aromatase mRNA expression levels were higher in breast cancer patients who developed local recurrence and distal metastasis [23]. The high levels of aromatase mRNA expression in non-cancer patients, therefore, gave us a hint of what might happen to the benign breast cancers. Biomarkers for risk assessment using an intraductal approach have been discussed recently [24-26] and Q-PCR provides a clinically acceptable technique for biomarker research. This offers a way to verify whether the aromatase level in NAF atypia is a predictive factor for future clinical management. A cohort study of the FNA benign breast patients is needed to confirm it.

Our research showed that the aromatase mRNA expression level in post-menopausal women was generally high compared with the level in pre-menopausal women; a similar association was not observed from the steroid sulfatase expression level $(P=0.529)$. This result might be because of the small specimen number that was available for the steroid sulfatase test. The enzyme expression levels were analyzed in the cancer and non-cancer groups; surprisingly, we found no relationship between steroid sulfatase mRNA expression levels and cancer incidence, contradicting the results of other studies $[27,28]$. It is possible that race or different sample collecting methods may explain this difference.

Estrogen action is mediated by ER receptors and the presence of $\mathrm{ER} \alpha$ has been implicated in a better prognosis for response to hormonal therapy [29]. ER status was absent in the clinical parameters because the FNA patients with benign lesions went home without surgery. The ER $\alpha$ mRNA expression level was analyzed to verify the association between the enzymes and ER mRNA expression. Our results showed no linear relationship between the ER and enzymes mRNA expression levels.

The present study had several drawbacks. Patient numbers were small and the ages of the patients in the cancer and non-cancer groups did not match; in particular, there was a lack of post-menopausal women in the non-cancer group. Therefore, the conclusions from this study need further verification in a larger sample population.

In summary, we tested aromatase and steroid sulfatase mRNA expression levels of FNA specimens by a nested Q-PCR assay. Our results suggested that both nested and non-nested Q-PCR assay were effective methods for analyzing breast FNA samples. We found that age and aromatase mRNA expression levels were two independent risk factors for breast cancer.

This work was supported by the Guang An'men Hospital, China Academy of Chinese Medical Sciences (2011S244). We thank Professor Richard J. Santen for help in correcting this paper.

1 Budtz P E. Role of proliferation and apoptosis in net growth rates of human breast cancer cells (MCF-7) treated with oestradiol and/or ta- 
moxifen. Cell Prolif, 1999, 32: 289-302

2 Yue W, Wang J P, Hamilton C J, et al. In situ aromatization enhances breast tumor estradiol levels and cellular proliferation. Cancer Res, 1998, 58: 927-932

3 Henderson B E, Ross R, Bernstein L. Estrogens as a cause of human cancer: The Richard and Hinda Rosenthal Foundation award lecture. Cancer Res, 1988, 48: 246-253

4 Pasqualini J R, Chetrite G, Blacker C, et al. Concentrations of estrone, estradiol, and estrone sulfate and evaluation of sulfatase and aromatase activities in pre- and post-menopausal breast cancer patients. J Clin Endocrinol Metab, 1996, 81: 1460-1464

5 Reed M J. The role of aromatase in breast tumors. Breast Cancer Res Treat, 1994, 30: 7-17

6 Zhang Z, Yamashita H, Toyama T, et al. Quantitative determination, by real-time reverse transcription polymerase chain reaction, of aromatase mRNA in invasive ductal carcinoma of the breast. Breast Cancer Res, 2003, 5: R250-R256

7 Locke I, Mitchell G, Eeles R. Ductal approaches to assessment and management of women at high risk for developing breast cancer. Breast Cancer Res, 2004, 6: 75-81

8 Liu G J, Wu Y S, Brenin D, et al. Development of a high sensitivity, nested Q-PCR assay for mouse and human aromatase. Breast Cancer Res Treat, 2008, 111: 343-351

9 Khan S A, Bhandare D, Chatterton R T, et al. The local hormonal environment and related biomarkers in the normal breast. Endocr Relat Cancer, 2005, 12: 497-510

10 Petrakis N L. ASPO Distinguished Achievement Award Lecture. Studies on the epidemiology and natural history of benign breast disease and breast cancer using nipple aspirate fluid. Cancer Epidemiol Biomarkers Prev, 1993, 2: 3-10

11 Bertero C, Chamberlain W M. Breast cancer diagnosis and its treatment affecting the self: A meta-synthesis. Cancer Nurs, 2007, 30: 194-202

12 Russo J, Hasan L M, Balogh G, et al. Estrogen and its metabolites are carcinogenic agents in human breast epithelial cells. J Steroid Biochem Mol Biol, 2003, 87: 1-25

13 Leong S P, Shen Z Z, Liu T J, et al. Is breast cancer the same disease in Asian and western countries? World J Surg, 2010, 34: 23082324

14 Kulendran M, Salhab M, Mokbel K. Oestrogen-synthesising enzymes and breast cancer. Anticancer Res, 2009, 29: 1095-1109

15 Bulun S E, Price T M, Aitken J, et al. A link between breast cancer and local estrogen biosynthesis suggested by quantification of breast adipose tissue aromatase cytochrome P450 transcripts using competitive polymerase chain reaction after reverse transcription. J Clin En- docrinol Metab, 1993, 77: 1622-1628

16 Tekmal R R, Ramachandra N, Gubba S, et al. Overexpression of int-5/aromatase in mammary glands of transgenic mice results in the induction of hyperplasia and nuclear abnormalities. Cancer Res, 1996, 56: 3180-3185

17 Kinoshita Y, Chen S. Induction of aromatase (CYP19) expression in breast cancer cells through a nongenomic action of estrogen receptor alpha. Cancer Res, 2003, 63: 3546-3555

18 Chen S. Aromatase and breast cancer. Front Biosci, 1998, 3: 922-933

19 London S J, Connolly J L, Schnitt S J, et al. A prospective study of benign breast disease and the risk of breast cancer. JAMA, 1992, 267: 941-944

20 Shekhar P V, Werdell J, Basrur V S. Environmental estrogen stimulation of growth and estrogen receptor function in preneoplastic and cancerous human breast cell lines. J Natl Cancer Inst, 1997, 89: 1774-1782

21 Salhab M, Reed M J, Al Sarakbi W, et al. The role of aromatase and 17-beta-hydroxysteroid dehydrogenase type $1 \mathrm{mRNA}$ expression in predicting the clinical outcome of human breast cancer. Breast Cancer Res Treat, 2006, 99: 155-162

22 Bollet M A, Savignoni A, De Koning L, et al. Tumor aromatase expression as a prognostic factor for local control in young breast cancer patients after breast-conserving treatment. Breast Cancer Res, 2009, 11: R54

23 Cazzaniga M, Decensi A, Bonanni B, et al. Biomarkers for risk assessment and prevention of breast cancer. Curr Cancer Drug Targets, 2009, 9: 482-499

24 Evans T R, Rowlands M G, Law M, et al. Intratumoral oestrone sulphatase activity as a prognostic marker in human breast carcinoma. Br J Cancer, 1994, 69: 555-561

25 Utsumi T, Yoshimura N, Takeuchi S, et al. Steroid sulfatase expression is an independent predictor of recurrence in human breast cancer. Cancer Res, 1999, 59: 377-381

26 Utsumi T, Yoshimura N, Takeuchi S, et al. Elevated steroid sulfatase expression in breast cancers. J Steroid Biochem Mol Biol, 2000, 73: 141-145

27 Newman S P, Purohit A, Ghilchik M W, et al. Regulation of steroid sulphatase expression and activity in breast cancer. J Steroid Biochem Mol Biol, 2000, 75: 259-264

28 Irahara N, Miyoshi Y, Taguchi T, et al. Quantitative analysis of aromatase, sulfatase and 17beta-HSD(1) mRNA expression in soft tissue metastases of breast cancer. Cancer Lett, 2006, 243: 23-31

29 Ali S, Coombes R C. Estrogen receptor alpha in human breast cancer: Occurrence and significance. J Mammary Gland Biol Neoplasia, 2000, 5: 271-281

Open Access This article is distributed under the terms of the Creative Commons Attribution License which permits any use, distribution, and reproduction in any medium, provided the original author(s) and source are credited. 